\title{
FRACTAL-BASED SPATIAL DISTRIBUTION ANALYSIS OF GEOLOGICAL HAZARDS AND MEASUREMENT OF SPATIAL ASSOCIATION WITH HAZARD-RELATED PREDISPOSING FACTORS
}

\author{
Q. Hu ${ }^{1,2,3}$, Y. Zhou ${ }^{2,3, *}$, S. X. Wang ${ }^{2,3}$, F. T. Wang ${ }^{2,3}$, H.J. Wang ${ }^{1,2,3}$ \\ ${ }^{1}$ University of Chinese Academy of Sciences, Beijing, China - (huqiao, wanghj)@radi.ac.cn \\ ${ }^{2}$ Aerospace Information Research Institute, Chinese Academy of Sciences, Beijing, China - zhouyi@radi.ac.cn, wangft@aircas.ac.cn \\ ${ }^{3}$ Institute of Remote Sensing and Digital Earth, Chinese Academy of Sciences, Beijing, China - WangSX@irsa.ac.cn
}

Commission VI, WG VI/4

KEY WORDS: Fractal dimension, Landslide, Collapse, Mudslide, Predisposing factor, China

\begin{abstract}
:
Fractal model as an effective solution to complex nonlinear problems or phenomena has been widely used to describe such complicated phenomenon as geological hazards. Quantitative analysis of the spatial distribution characteristics of geological hazards and measuring its fractal relation on a national scale are significant for the geological hazards prevention or mitigation. In this contribution, firstly, three typical geological hazards, such as landslides, collapses and mudslides, were taken as research objects for fractal analysis, and a detailed hazard inventory including 109,008 landslides, 55,178 collapses, and 28,914 mudslides cases were compiled as data samples. Next, the fractal dimensions describing the spatial distribution characteristics of geological hazard densities were calculated by the invariant fractal model, and then the internal classification of five common predisposing factors (elevation, slope, aspect, NDVI, and precipitation) was applied, and the relative density of geological hazard was calculated by the ratio of "hazard ratio" and "grid ratio" on the basis of $1 \mathrm{~km} \times 1 \mathrm{~km}$ grid cells. Finally, the variable fractal model was introduced for measuring the spatial association among three typical geological hazards and five common predisposing factors, and the obtained fractal dimensions were regarded as the quantitative measure of the effect of predisposing factors on geological hazards. The results shows that the fractal dimensions of spatial distribution of landslide, collapse and mudslide densities are 1.3042, 1.5185 and 1.5897, respectively. Moreover, the relative densities of geological hazards also follows the fractal features with hazard-related predisposing factors, the elevation factor has the greatest impact on the landslide, collapse, and mudslide hazard, while other predisposing factors have different effects on different types of geological hazards.
\end{abstract}

\section{INTRODUCTION}

Landslides, collapses and mudslides are the most common geological hazards worldwide and they cause enormous casualties and severe economic losses every year (Guzzetti et al., 2012; Pradhan et al., 2016). In China, especially the southeast coastal, middle-south, and southeast areas have been seriously affected by landslides, collapses and mudslides, which pose a serious threat to the environment, residential districts, and industrial facilities (Xu et al., 2015).

Hazard susceptibility mapping aims to express the predicted results of where landslides are likely to occur on the basis of local geographical conditions (Guzzetti et al., 2005) and there is consensus among managers that they can be used to effectively assess and respond to geological hazards (Hong et al., 2016). Analyzing the spatial distribution characteristics of geological hazards and measuring the spatial relationship between hazards and hazard-related predisposing factors are essential to understand and recognize the key conditioning features of hazard formation in order to produce reliable susceptibility map (Bui et al., 2011).

Over the past several decades, although the development of various methods, such as the information value, weights of evidence, frequency ratio, Dempster-Shafer method, certainty factors, logistic regression, Bayesian algorithms (e.g. NB, Bayesian networks), Support vector machines, artificial neural networks, tree models and fractal model (Li et al., 2012; Zuo et al., 2017) all have proposed to analyze the characteristics of predisposing factors related to geological hazards for quantitative assessment of geological hazards susceptibility, the common of these methods or models mentioned above are to

\footnotetext{
* Corresponding author
}

first analyze the potential association between geological hazards and predisposing factors, so as to determine the impact of geo-environment factors on hazards.

Compared with other nonlinear methods, fractal model is more robust because fractal dimension can be the fraction, which greatly enriches the traditional theories whose dimension is the integer (Mandelbrot. 1967, Lu et al., 2012). The fractal or multifractal model has been widely applied to provide an effective solution to complex nonlinear problems or phenomena, such as geological hazards (Li et al., 2011, 2012; $\mathrm{Lu}$ et al., 2012). In current studies, fractal models are mainly applied to investigate hazards' characteristics in the following aspects (Zuo et al., 2017), $i$. the frequency-area statistics of landslides (Ghosh et al., 2012b; Trigila et al., 2010; Turcotte and Malamud, 2004; Guzzetti et al., 2002); ii. the frequency-size statistics of landslides (Malamud et al., 2004; Iwahashi et al., 2003; Pelletier et al., 1997); iii. the cumulative frequency statistics of landslides ( $\mathrm{Li}$ et al., 2011), and $i v$. statistics of the landslide spatial distribution characteristics at different scales(Rouai and Jaaidi, 2003). However, the question of how to evaluate the relationship between geological hazards and predisposing factors from a fractal perspective is not well explored (Zuo et al., 2017). In particular, the use of fractal models to study the characteristics of geological hazards on a national scale is also still lacking in China.

In this study, based on 251943 geological hazards $(109,008$ landslides, 55,178 collapses and 28,914 mudslides) location information which are recorded nationwide, fractal models were introduced to analyze and measure the spatial distribution characteristics and spatial association among three typical geological hazards (landslides, collapses and mudslides) and five common predisposing factors such as elevation, slope, aspect, NDVI, and precipitation. To our knowledge, our work 
differs from previous studies is that we first introduce fractal model to evaluate the hazard characteristics on a national scale.

\section{DATA USED}

\subsection{Geological Hazard Inventory}

In this study, geological hazard records were collected from "GeoCloud", an online geological database (http://geocloudsso.cgs.gov.cn/) published by China Geological Survey. All historical hazards information showed on "GeoCloud" were compiled through on-site measures and field surveys of local administrations. The detailed information contained in this hazards inventory comprises the hazard name, hazard type, occurrence location, hazard levels, structural positions, groundwater type, seismic intensity, leading edge elevation, trailing edge elevation, economic loss, population casualties, management status, and so on. Finally, according to the criteria in Table 1, these information of 109,008 landslides (412 super large landslides, 5566 large landslides, 25392 medium-size landslides, and 77638 minor landslides), 55,178 collapses (1074 super-large collapses, 1388 large collapses, 9297 medium-size landslides, and 43419 minor landslides) and 28,914 mudslides (458 super-large collapses, 2302 large collapses, 6458 medium-size landslides, and 19696 minor landslides) locations (centroids) in China were identified as sample data for further analysis.

\begin{tabular}{llll}
\hline Classification & $\begin{array}{l}\text { Landslide } \\
\left(10^{4} \mathrm{~m}^{3}\right)\end{array}$ & $\begin{array}{l}\text { Collapse } \\
\left(10^{4} \mathrm{~m}^{3}\right)\end{array}$ & $\begin{array}{l}\text { Mudslide } \\
\left(10^{4} \mathrm{~m}^{3}\right)\end{array}$ \\
\hline Super-large & $\geq 1000$ & $\geq 100$ & $\geq 50$ \\
Large & $100 \sim 1000$ & $10 \sim 100$ & $20 \sim 50$ \\
Medium-size & $10 \sim 100$ & $1 \sim 10$ & $2 \sim 20$ \\
Minor & $<10$ & $<1$ & $<2$ \\
\hline
\end{tabular}

Table 1. Scale division of landslides, collapses and mudslides

\subsection{Predisposing factors}

Due to the regional differences of geographical environment, the types and mechanisms of geological hazards in different areas are very complex, and the type of hazard-related predisposing factors may have been conditioned by the local setting and the geo-environmental features. Conditional factors for describing morphology such as elevation, slope and aspect have proven particularly effective in predicting the spatial distribution of geological hazards (Fabbri et al., 2003), so in this study, the relationship between hazard-related common factors such as elevation, slope and aspect and geological hazards were considered.

Elevation, slope, and aspect are the typical variables used to describe morphology (Kalantar et al. 2017) and always obtained from the DEM data. In this study, DEM data with resolution of $30 \mathrm{~m} \times 30 \mathrm{~m}$ was derived from the National Basic Science Data Sharing Service Platform, Chinese Academy of Sciences (http://www.gscloud.cn). Elevation is affected by geomorphological and geological processes, terrain slope controls the balance of the retaining and the destabilizing forces acting on a slope, and a larger resistance is mobilized to maintain stable a steep slope than a gentle slope. Slope aspect has a crucial effect on hazards because weathering is affected by exposure to sunlight, winds, and precipitation (Kalantar et al. 2017; Ding et al. 2017).

NDVI is a quantitative parameter of vegetation coverage and reflects ecological environmental quality. It can directly affect the degree of soil erosion and the modification of the slope surface (Du et al. 2017). The formation of plant root complexes in the surface soil can help maintain slope stability by enhancing the shear strength of slope soil (Wang et al. 2017; Huang et al. 2017). Annual average NDVI data were calculated from the spatial distribution dataset of China's annual NDVI (2010-2018) (http://www.resdc.cn/DOI/doi.aspx?DOIid=49).

Moreover, the spatial and temporal distribution of precipitation is not uniform. During precipitation infiltration, liquefaction of the soil causes a gradual decrease in the material suction, which leads to a decrease of shear strength and induces landslides (Pham et al., 2017; Duc, 2012). The precipitation data were derived from the data set of surface climate data provided by the Resource and Environment Data Cloud Platform, Chinese Academy of Sciences (http://www.resdc.cn/).

\section{METHOD}

\subsection{Invariant Fractal model}

Fractal model was first introduced by Mandelbrot and has become a new method to study such complicated phenomenon as earthquake and geological hazards in recent years (Ge et al., 2018). The fractal model can be described as a power-law expressed by Eq. (1) (Li et al., 2012). When $\ln (l)$ and $\ln (r)$ satisfy the linear fitting characteristic, $D$ is a fixed value and which means the invariant fractal dimension.

$$
\begin{aligned}
& l(r)=\mathrm{C} \times \mathrm{r}^{-\mathrm{D}} \\
& \Rightarrow \ln (l(r))=-D \ln (r)+\ln (C)
\end{aligned}
$$

where $r=$ feature measured scale

$l=$ the measured value under the corresponding scale $r$

$D=$ the fractal dimension

$C=$ a constant

In this study, the invariant fractal method was used to analyze the spatial distribution characteristics of geological hazards. We consider the hazards in a region as a set of points in the two-dimensional space. For the study region, we discretize it into square grid cells of different size and count the number of cells that contain at least one hazard corresponding to grid unit size. Next, the density of geological hazards were calculated at different grid scales. Finally, the measurement scale and the corresponding hazard density values were used as $l$ and $r$ substituting Eq. 1, respectively, for double logarithmic fitting and fractal dimension calculation.

\subsection{Variable Dimension Fractal Model}

Usually in many study, the invariant fractal relationship does not strictly exist in nature, so $\ln (l)$ and $\ln (r)$ cannot be well fitted linearly in the case of some sophisticated phenomena $(\mathrm{Lu}$ et al., 2012). Therefore, the application of the traditional invariable fractal dimension method is limited. In many practical applications, as Newman (2005) and Li et al., (2012) pointed out, one of the methods of studying the data is to calculate the cumulative distribution function. The cumulative sum can be calculated by Eq. (2).

$$
\begin{aligned}
& L(r)=l(R \leq r)=\int_{r_{\min }}^{r} l\left(r^{\prime}\right) d r^{\prime} \\
& =C \int_{r_{\min }}^{r} r^{\prime-D} d r^{\prime}=\frac{C}{D-1} r^{-(D-1)}
\end{aligned}
$$

where $\quad L(r)=$ the cumulative sum of $l(r)$ 


\section{$R=$ a value less than $r$}

In this study, as an extension to applications of the power-law (fractal) distribution, the specific method mainly includes the following steps:

First, the internal classification of each predisposing factor was applied, and the hazard relative density $P$ was calculated by Eq.(3).

$$
P=P_{h d} / P_{g d}=\frac{\left(N_{h} / N\right)}{M_{g} / M}
$$

where $\quad N_{h}=$ the number of hazards in one predisposing factor subclass

$N=$ the total number of landslides

$P_{h d}=$ the ratio of $N_{h}$ to $N$

$M_{g}=$ the number of grid units in the same predisposing factor subclass

$M=$ the total number of grid units

$P_{g d}=$ the ratio of $M_{g}$ to $M$.

$P=$ the relative density of geological hazards

Second, each subclass is numbered $0,1,2, \ldots$ in descending order of $P$ values as the feature measured scale $r$. If the double logarithmic curve of the raw data points $(P, r)$ cannot be linearly fitted (the $R^{2}$ value of the fitting curve would be above 0.95 ), then the cumulative sum (S) of $P$ can be constructed as Eq.(4) (Lu et al., 2012; Li et al., 2011, 2012). Next, the data points $(S, r)$ are plotted on the double logarithmic coordinates, and linear fitting is carried out to obtain the variable dimension fractal model.

$$
\begin{aligned}
& \{P\}=\left\{P_{1}, P_{2}, \cdots \cdots P_{\mathrm{n}}\right\} \\
& \left\{S_{1}\right\}=\left\{P_{1}, P_{1}+P_{2}, \cdots \cdots, P_{1}+P_{2}+P_{3}+\cdots P_{\mathrm{n}}\right\} \\
& \left\{S_{2}\right\}=\left\{S_{11}, S_{11}+S_{12}, \cdots \cdots, S_{11}+S_{12}+S_{13} \cdots+S_{1 n}\right\} \\
& \left\{S_{3}\right\}=\left\{S_{21}, S_{21}+S_{22}, \cdots \cdots, S_{21}+S_{22}+S_{23} \cdots S_{2 n}\right\}
\end{aligned}
$$

where $\quad S_{i}=$ the $i$-th order cumulative sum of $P$.

\section{RESULTS AND DISCUSSION}

\subsection{Spatial Distribution of Geological Hazard Density}

In order to explain this hazard densities distribution behavior using the fractal concept, landslide, collapse and mudslide hazards occurred in China over the years and were taken as data samples, and $1 \times 1 \mathrm{~km}, 1.5 \times 1.5 \mathrm{~km}, 2 \times 2 \mathrm{~km}, \ldots$, and $10 \times 10$ $\mathrm{km}$ regular grid models covering the whole of China are respectively established at the size intervals of $0.5 \mathrm{~km}$, and then the number of grid units and the number of grid units containing geological hazards were counted at different grid scales, and then the hazard densities of three kinds of geological hazards were also calculated separately. Each the hazard density distribution curves were drawn in a double logarithmic plot (Figure. 1). In these three plots, the high root-square $\left(\mathrm{R}^{2}\right)$ value reveals that the log-log plots of hazard density versus the scale of grid unit can be fitted with straight lines by the least-squares method. From a spatial statistic point, the size of grid units do not influence the slope of the log-log plot of hazard density, and therefore, the resulting fractal dimension could not change when different grid unit sizes were used. Moreover, it was observed that the larger the size of grid unit, the greater the density of three geological hazards. This phenomenon suggests a nonlinear spatial distribution of hazard density, and meaning that hazard densities can be expressed as a power-law function of measurement scales. In addition, in terms of the three hazard densities, the values of three fractal dimensions are between 0 and 2, this results show that the fractal clustering distribution pattern of landslide, collapse and mudslide densities are different from random distributions (e.g., Poisson distribution) and also different from the uniform distribution (Li et al., 2012). Comparing the fractal dimensions of these geological hazard densities, mudslide density has the largest (1.5897) fractal dimension, followed by the collapses (1.5185) and landslides (1.3042), it suggests that the spatial clustering of mudslides density is more obvious than that of collapses and landslides.

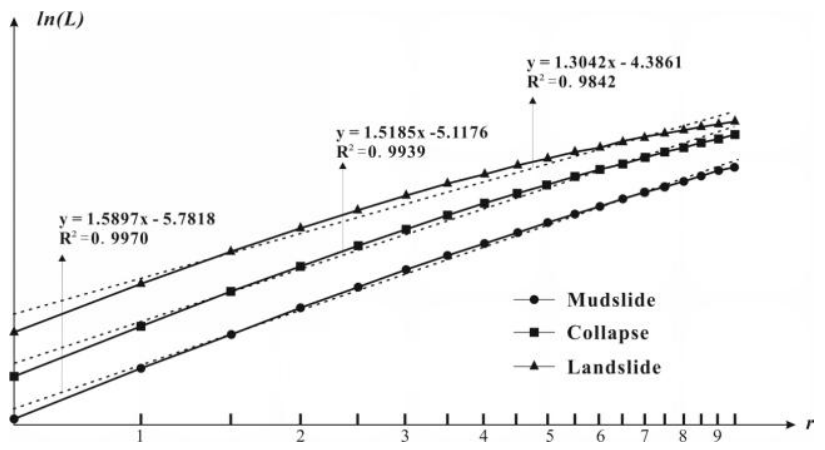

Figure 1. Fractal analysis of density of geological hazards

\subsection{Fractal Relationship between Geological Hazards and Predisposing factors}

Elevation, slope, aspect, NDVI, precipitation are the most widely used hazard-related predisposing factors in geological hazard study. It is of great significance to analyze the relationship between these common geo-environmental factors and geological hazards on a national scale and to evaluate the influence weight of these predisposing factors on hazards in order to sort out the causes of disasters.

In view of the large scope of the research area, these hazard-related predisposing factors with different scales were calibrated to the uniform grid unit size of $1 \times 1 \mathrm{~km}$, and the equidistant classification method was used for the internal classification of five applied predisposing factors. Then, the factors of elevation, slope, NDVI, and precipitation were divided into eleven subcategories and aspect factor was divided into nine subcategories for further analysis.

As shown in Figure 2, the 1-order variable dimensional fractal relationship exists in elevation and collapse, elevation and collapse, NDVI and landslide, NDVI and collapse, NDVI and mudslide, precipitation and landslide, precipitation and mudslide. In the remaining cases, the relationship between the predisposing factors and the hazards satisfies the 0 -order fractal, this also shows that they meet the power law distribution characteristics. 


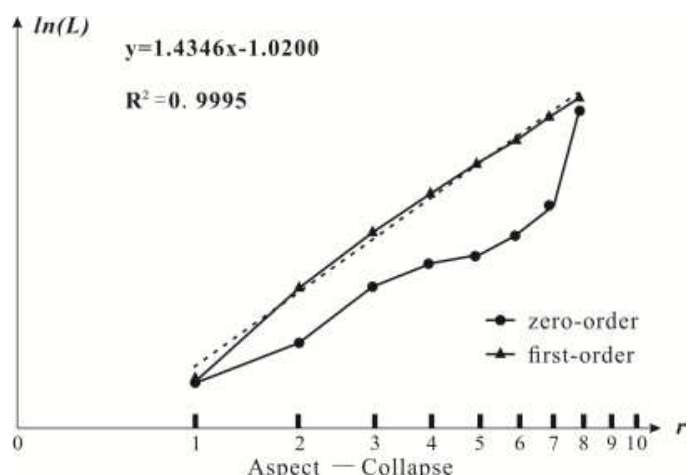

\section{$\uparrow \ln (L) \quad \mathrm{y}=1.4427 \mathrm{x}-1.0521$}

$R^{2}=0.9968$
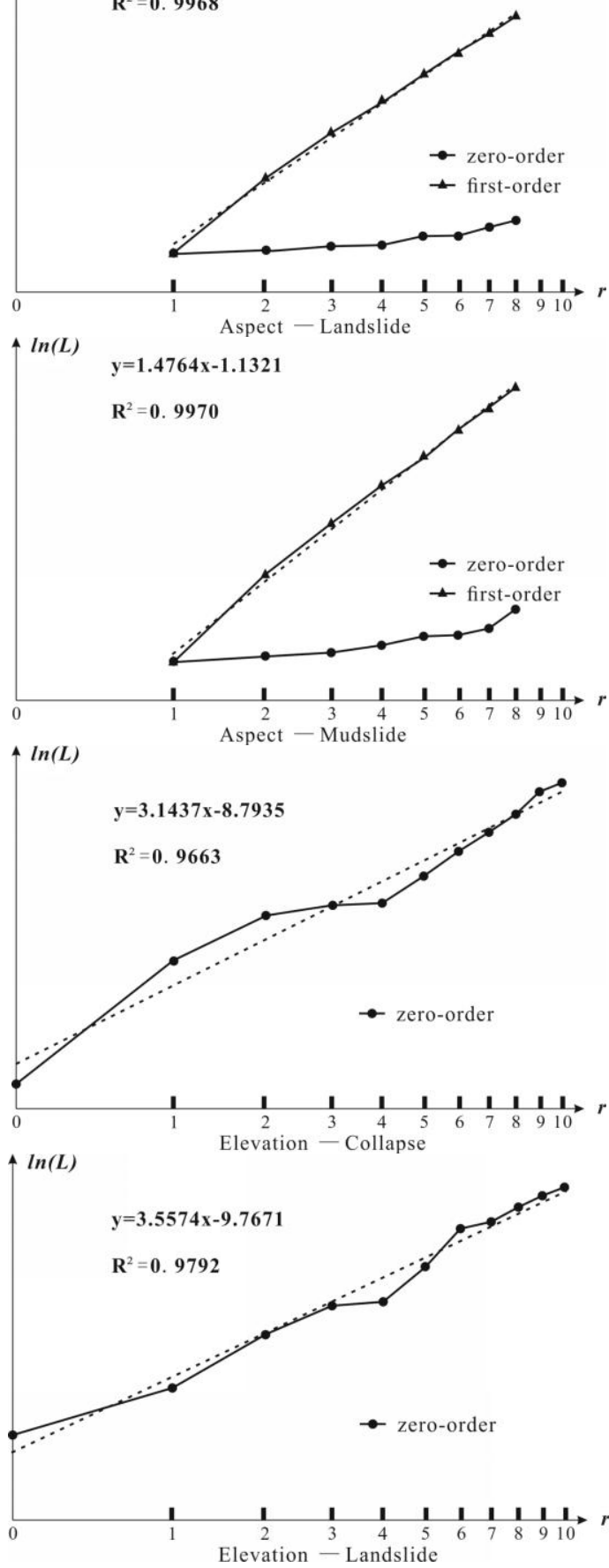
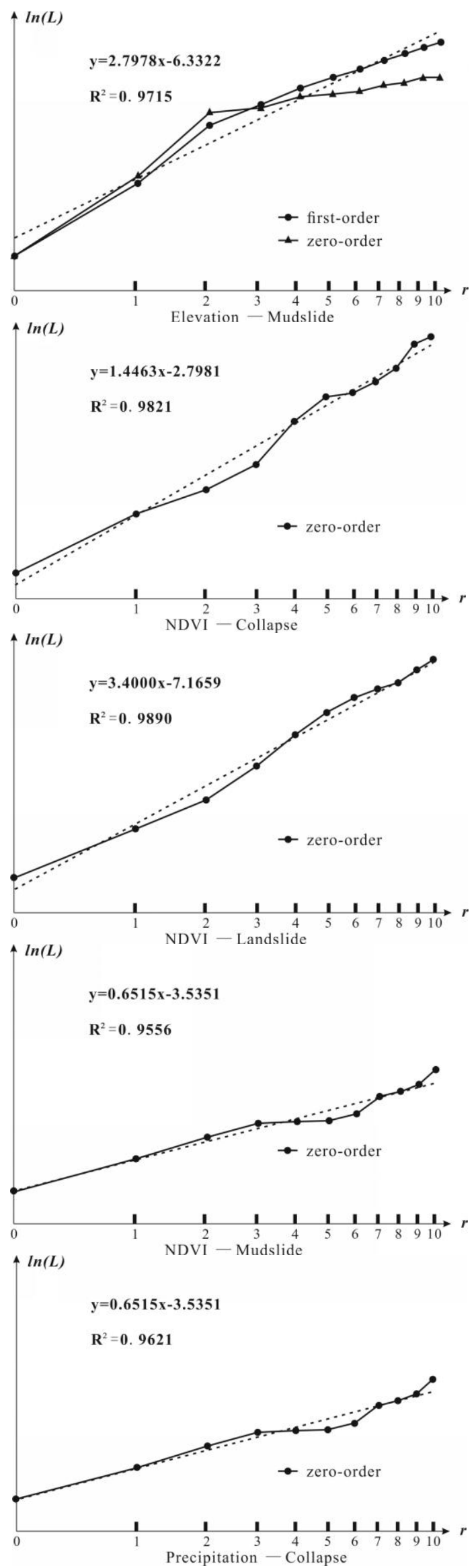

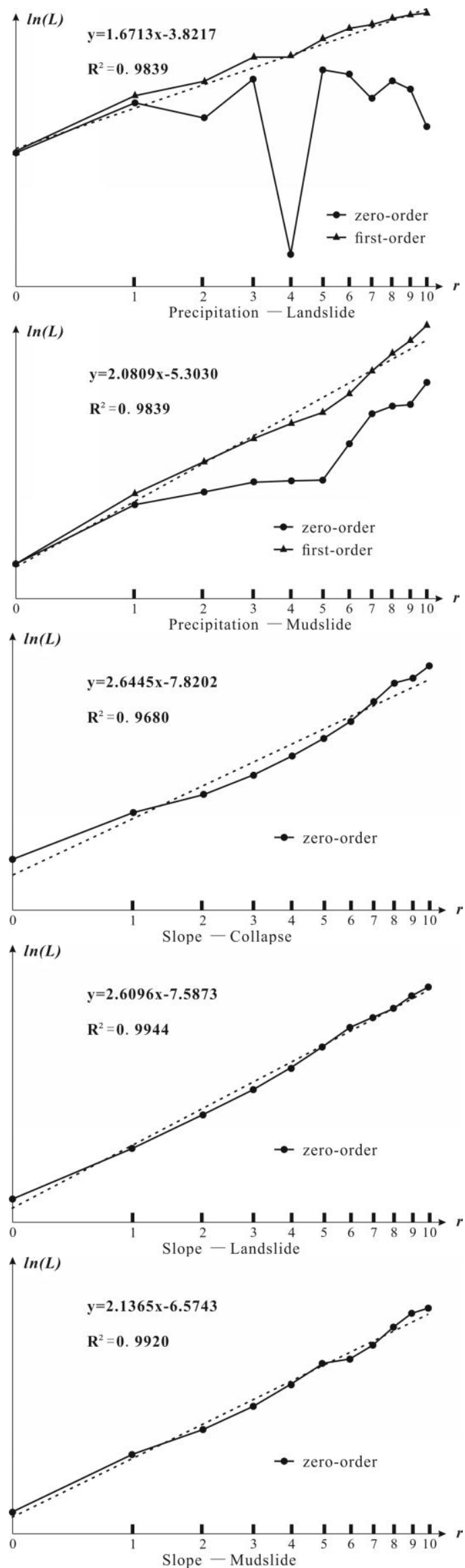

Figure 1. Fractal analysis between geological hazards and predisposing factors
According to Zuo et al. (2012) and Ge et al. (2018), the fractal dimension can represents a significant spatial association between a specific predisposing factor and corresponding geological hazards. In this study, the acquired fractal dimension (Table 2) of five predisposing factors with landslides, collapses, and mudslides were used as weights of each factor on hazard occurrence. Then, on the basis of the unified standard condition $\mathrm{R}^{2} \geq 0.95$, we use straight lines to fit the highest order curve in the double logarithmic coordinate linearly, and the fractal dimension value of each predisposing factor directly estimated from the slope of the fitted straight line. As far as landslide hazards are concerned, the fractal dimensions describing the correlation between five common predisposing factors and the relative density of landslide hazards were $3.557,2.610,1.443$, 3.400 , and 1.671 , respectively. This means that the influence of elevation on landslides is the greatest, and followed by NDVI, slope, precipitation, and aspect.

In term of collapses, elevation factor is also the most important topographic factor affecting collapse hazards, the fractal dimension between elevation factor and relative density of collapses is 3.144 , followed by the predisposing factors of slope (2.645), NDVI (1.446), aspect (1.435), and precipitation (0.652). With regard to mudslides, the fractal dimension of elevation predisposing factor is still the highest, which value of fractal dimension is 2.798 , and the predisposing factor of slope had the more influence, the corresponding fractal dimension is 2.137 , then the fractal dimension of precipitation and aspect are 2.081 and 1.476, respectively. Moreover, the predisposing factor of NDVI achieves the minimum fractal dimension (1.220), suggesting that the NDVI play a less important role in the mudslides than other predisposing factors.

\begin{tabular}{llll}
\hline Fractal dimension & Landslide & Collapse & Mudslide \\
\hline Elevation & 3.557 & 3.144 & 2.798 \\
Slope & 2.610 & 2.645 & 2.137 \\
Aspect & 1.443 & 1.435 & 1.476 \\
NDVI & 3.400 & 1.446 & 1.220 \\
Precipitation & 1.671 & 0.652 & 2.081 \\
\hline
\end{tabular}

Table 2. Fractal dimension of predisposing factors

On the whole, among the five hazard-related predisposing factors selected in this study, elevation has the greatest impact on these three typical geological hazards, especially on landslides, which has greater impact than collapses and mudslides. For slope factor, the influence of slope on collapses and mudslides is second only to that of elevation on collapses and mudslides, but the influence of slope on landslides is much smaller than that of NDVI on landslides, this shows that vegetation cover has more obvious control effect on landslides than terrain slope. However, the impact of NDVI on mudslides is quite different from that on landslide hazards, which also shows that landform factors have less impact on mudslide disasters. Rainfall, the only meteorological factor considered, has a great impact on mudslides, this features are consistent with the occurrence mechanism of mudslide hazards, because heavy rainfall is one of the necessary conditions leading to mudslide hazards, but for landslides and collapses, the effects of precipitation on landslides and collapses are relatively weak, which may be due to the diversity of landslides, while the cases of rainfall-type landslides are less distribute in the hazard inventory used in study.

\section{CONCLUSIONS}

In this study, based on the invariant fractal model, the fractal 
characteristics of the spatial distribution of landslide, collapse, and mudslide densities in whole China were analyzed and calculated the fractal dimension of these three common geological hazards. Moreover, the variable fractal model was used for measuring the spatial relationships between five typical predisposing factors (elevation, slope, aspect, NDVI, and precipitation) and these three common geological hazards (landslides, collapses, and mudslides). The following conclusions are obtained: (1) the spatial of the densities of landslides, collapses, and mudslides satisfies the invariant fractal characteristics, the fractal dimension of the densities of landslides, collapses, and mudslides are 1.3042, 1.5185 and 1.5897 , respectively; (2) relative density of geological hazards follows a variable fractal relation with hazard-related predisposing factors such as elevation, slope, aspect, NDVI, and precipitation; (3) the fractal dimension is a robust parameter for measuring the relative importance of conditioning factors of hazard occurrence, and can provides critical information for hazard susceptibility assessment or mapping, so as to prevent and/or mitigate geological hazards; and (4) the calculated fractal dimension suggested that the elevation factor has the greatest impact on the landslides, collapses, and mudslides, while other predisposing factors have different effects on different types of geological hazards.

\section{ACKNOWLEDGMENTS}

This research was supported by the National Key Research and Development Program of China (2017YFB0504100, 2016YFC0803000) and Youth Innovation Promotion Association of the Chinese Academy of Sciences (2015129).

\section{REFERENCES}

Ding, Q.F., Chen, W., Hong, H.Y., 2017. Application of frequency ratio, weights of evidence and evidential belief function models in landslide susceptibility mapping. Geocarto International 32(6), 619-639. doi.org/10.1080/10106049.2016.1165294

Du, G.L., Zhang, Y.S., Iqbal, J., Yang, Z.H., Yao, X., 2017. Landslide susceptibility mapping using an integrated model of information value method and logistic regression in the Bailongjiang watershed, Gansu Province, China. Journal of Mountain Science 14(2), 249-268. doi.org/10.1007/s11629-016-4126-9

Duc, D.M., 2012. Rainfall-triggered large landslides on 15 December 2005 in Van Canh District, binh Dinh Province, Vietnam. Landslides 10 (2), 219-230. doi.org/10.1007/s10346-012-0362-4

Fabbri, A.G., Chung, C.-J.F., Cendrero, A., Remondo, J., 2003. Is prediction of future landslides possible with a GIS? Natural Hazards 30 (3), 487-503. doi.org/10.1023/b:nhaz.0000007282.62071.75

Ge, Y.F., Chen, H.Z., Zhao, B.B., Tang, H.M., Lin, Z.S., Xie, Z.G., Lv, L., Zhong, P., 2018. A comparison of five methods in landslide susceptibility assessment: a case study from the $330-\mathrm{kV}$ transmission line in Gansu Region, China. Environmental Earth Sciences 77(19), 662. doi.org/10.1007/s12665-018-7814-7

Ghosh, S., Carranza, E. J. M., 2010. Spatial analysis of mutual fault/fracture and slope controls on rocksliding in Darjeeling Himalaya, India. Geomorphology 122(1/2), 1-24.

\section{doi.org/10.1016/j.geomorph.2010.05.008}

Guzzetti, F., Mondini, A.C., Cardinali, M., Fiorucci, F., Santangelo, M., Chang, K.T., 2012. Landslide inventory maps: new tools for an old problem. Earth Science Reviews 112(1-2), 42-66.

doi.org/10.1016/j.earscirev.2012.02.001

Guzzetti, F., Reichenbach, P., Cardinali, M., Galli, M., Ardizzone, F., 2005. Probabilistic landslide hazard assessment at the basin scale. Geomorphology 72(1/2/3/4): 272-299.

doi.org/10.1016/j.geomorph.2005.06.002

Hong, H.Y., Naghibi, S.A., Pourghasemi, H.R., Pradhan, B., 2016. GIS-based landslide spatial modeling in Ganzhou City, China. Arabian Journal of Geosciences 9(2), 112. doi.org/10.1007/s12517-015-2094-y

Huang, F.M., Yin, K.L., Huang, J.S., Gui, L., Wang, P., 2017. Landslide susceptibility mapping based on self-organizing-map network and extreme learning machine. Engineering Geology $223,11-22$

doi.org/10.1016/j.enggeo.2017.04.013

Iwahashi, J., Watanabe, S., Furuya, T., 2003. Mean slope-angle frequency distribution and size frequency distribution of landslide masses in Higashikubiki area, Japan. Geomorphology 50(4): 349-364.

doi.org/10.1016/s0169-555x(02)00222-2

Kalantar, B., Pradhan, B., Naghibi, S.A., Motevalli, A., Mansor, S., 2017. Assessment of the effects of training data selection on the landslide susceptibility mapping: a comparison between support vector machine (SVM), logistic regression (LR) and artificial neural networks (ANN). Geomatics, Natural Hazards and Risk 9(1), 49-69.

doi.org/10.1080/19475705.2017.1407368

Li, C.J., Ma, T.H., Zhu, X.S., Li, W., 2011. The power-law relationship between landslide occurrence and rainfall level. Geomorphology 130(3/4): 221-229.

doi.org/10.1016/j.geomorph.2011.03.018

Li, C.J., Ma, T.H., Sun, L.L., Lei, W., Zheng, A.P., 2012. Application and verification of a fractal approach to landslide susceptibility mapping. Natural Hazards 61(1), 169-185. doi.org/s11069-011-9804-x

Lu, J., Wu, J., Yao, H., Qian, J.Z., Wang, Z.L., Wang, J.Q., 2012. Predicting river dissolved oxygen in complex watershed by using sectioned variable dimension fractal method and fractal interpolation. Environmental Earth Sciences 66(7), 2129-2135.

doi.org/10.1007/s12665-011-1437-6

Malamud, B.D., Turcotte, D.L., Guzzetti, F., Paola, R., 2004. Landslide inventories and their statistical properties. Earth Surface Processes and Landforms 29(6), 687-711. doi.org/10.1002/esp.1064

Mandelbrot, B., 1967. How long is the coast of Britain? statistical self-similarity and fractional dimension. Science 156(3775), 636-638.

doi.org/10.1126/science.156.3775.636

Newman, M.E.J., 2005. Power laws, Pareto distributions and Zipf's law. Contemporary Physics 46(5), 323-351. doi.org/10.1080/00107510500052444 
Pelletier, J.D., Malamud, B.D., Blodgett, T., Turcotte, D.L., 1997. Scale-Invariance of soil moisture variability and its implications for the frequency-size distribution of landslides. Engineering Geology 48(3/4), 255-268.

doi.org/10.1016/s0013-7952(97)00041-0

Pham, B.T., Bui, D.T., Dholakia, M.B., Prakash, I., Pham, H.V., Mehmood, K., Le, H.Q., 2017. A novel ensemble classifier of rotation forest and Naïve Bayer for landslide susceptibility assessment at the Luc Yen district, Yen Bai Province (Viet Nam) using GIS. Geomatics, Natural Hazard and Risk 8(2), 649-671. doi.org/10.1080/19475705.2016.1255667

Pradhan, A.M.S., Tarolli, P., Kang, H.S., Lee, J.S., Kim, Y.T., 2016. Shallow landslide susceptibility modeling incorporating rainfall statistics: a case study from the Deokjeok-ri Watershed, South Korea. International Journal of Erosion Control Engineering 9(1), 18-24. doi.org/10.13101/ijece.9.18

Rouai, M., Jaaidi, E. B., 2003. Scaling properties of landslides in the Rif mountains of Morocco. Engineering Geology 68(3/4), 353-359.

doi.org/10.1016/s0013-7952(02)00237-5

Trigila, A., Iadanza, C., Spizzichino, D., 2010. Quality assessment of the Italian landslide inventory using GIS processing. Landslides 7(4), 455-470.

doi.org/10.1007/s10346-010-0213-0

Turcotte, D. L., Malamud, B. D., 2004. Landslides, forest fires, and earthquakes: examples of self-organized critical behavior. Physica A-Statistical Mechanics and Its Applications 340(4), 580-589.

$$
\text { doi.org/10.1016/j.physa.2004.05.009 }
$$

Wang, Q., Wang, Y., Niu, R.Q., Peng, L., 2017. Integration of information theory, k-means cluster analysis and the logistic regression model for landslide susceptibility mapping in the Three Gorges Area, China. Remote Sensing 9(9), 938. doi.org/10.3390/rs9090938

Xu, C., Xu, X., Shu, J.B.H., 2015. Database and spatial distribution of landslides triggered by the Lushan, China $\mathrm{Mw}$ 6.6 earthquake of 20 April 2013. Geomorphology 248, 77-92. doi.org/10.1016/j.geomorph.2015.07.002

Zuo R.G., Carranza E.J.M. 2017. A fractal measure of spatial association between landslides and conditioning factors. Journal of Earth Science 28(4), 588-594. doi.org/10.1007/s12583-017-0772-2 